\title{
Die mehrgliedrige Kultur litoraler Folliculiniden ${ }^{1}$
}

\author{
Gotram UHLIG \\ Biologische Anstalt Helgoland, Meeresstation, Helgoland
}

\begin{abstract}
A multi-level culture of littoral folliculinids. Earlier experiments on folliculinids have been conducted primarily by employing the glass-plate method. The cultivation of sessile, loricated folliculinids under non-sterlile conditions is rather difficult because in culture dishes without circulating sea water an ever-thickening coat of film from detritus, bacteria and fungi builds up. Using a newly discovered harpacticid, Tisbe belgolandica n. sp., a multi-level culture method was developed. The euryplastic, ravenous harpaticids are extremely well suited to be "culture partners" of the folliculinids; they eat the detritusbacteria-fungi film and thus keep the cultures clean. By employing the food chain, Dunaliella and Cryptomonas $\rightarrow$ folliculinids $\rightarrow$ faeces $\rightarrow$ bacteria and fungi $\rightarrow$ Tisbe, a rather stable biological equilibrium can be achieved. Up until now this method has made it possible to cultivate successfully four littoral folliculinid species of the genera Metafolliculina, Eufolliculina and Diafolliculina. Method and handling of cultures are described in detail.
\end{abstract}

\section{EINLEITUNG}

Der ehrenwerte Nestor der Folliculinidenforschung, J. HaDžı, leitet den allgemeinen Teil seiner umfangreichen Monographie (1951) mit folgenden Sätzen ein: „Trotz der verhältnismäßig großen Anzahl veröffentlichter Werke ist unser Wissen über die Folliculiniden sehr mangelhaft. Namentlich gilt das für den Lebenskreis dieser niedlichen Tierchen (p. 24). "Unter anderem weist HADžı auf die Vernachlässigung einer „dauernden Züchtung unter normalen und experimentell geänderten Umständen“ hin. Es ist klar, daß eine Lösung der vielschichtigen Probleme, die sich aus dem recht ungewöhnlichen Lebenszyklus der Folliculiniden ergeben, nur nach erfolgreicher Kultivierung möglichst vieler Formen zu erhoffen ist. Die vergleichsweise wenigen, eingehenden Untersuchungen an lebenden Objekten wurden vornehmlich unter Anwendung der üblichen Glasplattenmethode durchgeführt (ANDREws 1914, SARHAGE 1916, Faure-Fremiet 1932, Dewey 1939, Hamilton 1952, Das 1953); man vermißt jedoch Angaben über weitergehende Kultivierungsexperimente.

Zweifellos bereitet die nicht-sterile Kultivierung sessiler Formen in Kulturschalen Schwierigkeiten besonderer Art: In wenigen Tagen bildet sich an der Schalenwandung zwangsläufig eine immer dichter werdende Schicht eines Detritus-Bakterien-Pilzgemisches, das die Gehäuse der Folliculiniden überwuchert und die Degeneration einer

${ }^{1}$ Herrn Professor Dr. Alfred Kühn zum 80. Geburtstag am 22. April 1965 in Verehrung gewidmet. 
Kultur bewirkt. Nach vielen Mißerfolgen lieferte der Zufall eine ebenso elegante wie natürliche Lösung dieses Problems. Die Entdeckung eines Harpacticiden - Tisbe helgolandica n. sp. - bildete die Basis für ein ausgewogenes, mehrgliedriges Kulturverfahren, das sich auch bei der Züchtung anderer, sedentärer Organismen bereits bewährt hat.

Bisher gelang es, vier Folliculiniden-Spezies aus dem Phytal in Kultur zu nehmen. Andere, noch nicht erprobte Flachwasserformen dürften in dieser Hinsicht keine wesentlichen Schwierigkeiten bereiten. Das Verfahren versagt allerdings bei sämtlichen Vertretern aus tieferen, küstenferneren Bereichen (Epifauna des Sandgrundes, siehe UhLig 1964b).

\section{DIE KULTIVIERTEN FORMEN}

\section{Metafolliculina andrewsi HAD ̌̌r 1938 (Abb. 1a)}

Die Gattung Metafolliculina ist nach Dons (1924) durch das Vorhandensein eines rosenkranzförmigen Makronucleus, relativ kleinen Wohnraum und hohen Hals mit kräftiger Spiralleiste gekennzeichnet. Bisher wurden dieser Gattung fünf Arten, $M$. andreweri, $M$. producta, $M$. longicollis, $M$. elongata und $M$. nordgardi beigeordnet. Die Trennung der vier erstgenannten Arten stützt sich namentlich auf eine unterschiedliche Halslänge der Gehäuse. Die noch laufenden Untersuchungen scheinen eine derartige Aufteilung nicht $\mathrm{zu}$ rechtfertigen. Offenbar fallen die gegebenen Artcharakteristika sämtlich in den Variabilitätsbereich von $M$. producta WRIGHT 1859. Vorbehaltlich einer endgültigen Bestätigung dieser These wird die hiesige Form vorerst als $M$. andrezsi geführt. Im Sommerhalbjahr ist $M$. andrewsi häufig als Epiphyt oder Epizoon in Helgoländer Felswatt vertreten.

$$
\text { Eufolliculina, zwei neue Arten (Abb. 1b) }
$$

Es handelt sich hier mit Sicherheit um zwei verschiedene Arten, deren Einordnung in das System noch nicht völlig geklärt ist. Von geringen Größenunterschieden abgesehen sind sich beide Arten morphologisch sehr ähnlich. Auffällig sind die unterschiedlichen Verhaltensweisen (Geotaxis, Thigmotaxis), die auch eine unterschiedliche Siedlungsweise bedingen. Die Tiere entstammen einem Seewasser-Aquarium, an dessen Wänden sie sich 1962 in großen Mengen angesiedelt hatten.

\section{Diafolliculina rotunda HADŽr 1951 (Abb. 1c)}

Im Sommerhalbjahr findet man $D$. rotunda recht häufig als Epiphyt auf verschiedenen Grün-, Rot- und Braunalgen. Die sehr kleine Form mit "Spatelfuß" trägt an der Halsbasis des Gehäuses (nur in der Seitenansicht sichtbar) zwei sehr zarte "Verschlußmembranen ". Das Peristom ist dorsal nur sehr schwach eingebuchtet und gleicht einem spiralförmigen Trichter. Herr Prof. J. HaDžI, Ljubljana (Jugoslawien), konnte 
die richtige Identifizierung dieser Form an Hand einer ihm zugesandten, lebenden Kultur bestätigen.

$$
\text { Dunaliella sp., Cryptomonas sp. }
$$

Eine nicht näher bestimmte Form von Dunaliella wird seit Jahren an der Biologischen Anstalt Helgoland in Reinkultur gehalten. Herr Prof. Grell, Tübingen, überließ mir 1961 einen Cryptomonas-Stamm, der sich auch für Fütterungszwecke gut bewährt hat.

$$
\text { Tisbe belgolandica n. sp. (Abb. 1d und e) }
$$

Im Frühjahr 1963 erbrachten verschiedene Kratzproben aus dem temperierten Helgoländer Seewasser-Freibad (etwa $22^{\circ} \mathrm{C}$ ) zwar nicht die erhofften thermophilen Folliculiniden, doch wurde ich durch einen interessanten und zugleich wertvollen Fund entschädigt. In den Uberlaufrinnen fand sich eine Vielzahl relativ großer Harpacticiden. Wie Herr Dr. Noodr, Kiel, feststellen konnte, handelt es sich um eine neue Art der Gattung Tisbe. Gegenwärtig wird diese Art als Tisbe belgolandica neu beschrieben. Dieser sehr gefräßige Harpacticide ist außerordentlich anspruchslos und weist bei entsprechendem Nahrungsangebot eine ungewöhnlich hohe Vermehrungsrate auf. Ein einziges Weibchen bildet in 20 Tagen bis zu 10 Eiersäcke, aus denen jeweils maximal etwa 80 Nauplien ausschlüpfen. Ein Tier kann demnach in etwa drei Wochen bis zu 800 Nauplien produzieren. Die frisclgeschlïpften Nauplien sind nach neun Häutungen (5 Naupliusstadien, 4 Copepoditstadien) innerhalb von 10 bis 14 Tagen geschlechtsreif. Der besondere Nutzen dieser Harpacticiden liegt darin, daß sie eine stark verschmutzte Kulturschale (Bakterien, feine Pilzhyphen, Detritus) innerhalb kurzer Zeit „abweiden“. In unseren Folliculiniden-Kulturen erwies sich diese Eigenschaft als besonders vorteilhaft.

\title{
KULTURTECHNIK
}

\section{Kulturmedien und Geräte}

\author{
Folliculiniden
}

Erprobt wurden einige modifizierte Erdschreiber-Lösungen sowie eine nach einfachem Rezept gefertigte, künstliche Seewasser-Lösung. Hinsichtlich der Wachstumsrate ergaben sich keine wesentlichen Unterschiede im Vergleich zu normalem Seewasser, so daß schließlich, der Vereinfachung wegen, nur filtriertes Seewasser verwendet wurde. Das Seewasser wird über eine SEITz-Filterscheibe (EK 5) direkt aus der Seewasserleitung in einen Ballon filtriert. Nach Abfüllung von jeweils 8 Liter in Jenaer Glasflaschen werden diese bei $90^{\circ} \mathrm{C}$ eine Stunde lang autoklaviert (gewissermaßen pasteurisiert). Auf diese Weise können kleinste Organismen, ohne Ausfällung bestimmter Salze, abgetötet werden. Kleine Kulturen (Klonkulturen) werden in Boverischalen 
angesetzt. Nach ausreichender Vermehrung können die Kulturen in größere Kulturschalen übertragen werden (siehe unten). Am besten bewährten sich sogenannte $\mathrm{Ab}$ dampfschalen (Höhe: $5 \mathrm{~cm}$, oberer Durchmesser: $10 \mathrm{~cm}$, mit oder ohne Ausguß), abgedeckt mit einem Uhrglas entsprechender Größe.

\section{Dunaliella; Cryptomonas}

Beide Formen werden nach altbewährter Methode in Erdschreiber-Lösung gezüchtet. Der Erdschreiber-Lösung wird eine Nitrat-Phosphat-Lösung (20 $\mathrm{g} \mathrm{NaNO}_{3}+$ $4 \mathrm{~g} \mathrm{NaHPO}_{4}$ auf $1000 \mathrm{ml} \mathrm{H} 2 \mathrm{O}$ ) zugesetzt, und zwar $5 \mathrm{ml}$ pro Liter "Erdschreiber". Für die Herstellung des Erdextraktes sei auf folgendes empfehlenswertes Verfahren hingewiesen: Etwa $100 \mathrm{~g}$ Erde werden in eine Extraktionshülse, $48 \times 200 \mathrm{~mm}$ (SCHLEICHER \& SCHüll Nr. 603) eingefüllt und mit Watte leicht abgedeckt. In einem SoxHLET-Extraktions-Apparat wird die Erde dann ungefähr sechs Stunden mit $750 \mathrm{ml}$ Aqua dest. extrahiert. Der so gewonnene Erdextrakt zeichnet sich durch seine hohe Transparenz aus. Das bisher erforderliche Absetzen, Dekantieren und Filtrieren einer Erdabkochung entfällt damit. Die Futtertiere werden in 5 Liter fassenden Enghalsflaschen gehalten, und zwar an der östlichen Fensterfront der Meeresstation Helgoland, unter Vermeidung direkter Sonneneinstrahlung. Es empfiehlt sich, stets nur frische Kulturen (log-Phase) als Futter zu verwenden.

Tisbe helgolandica

T. helgolandica stellt keine besonderen Ansprüche an ein Kulturmedium. Vorläufigen Untersuchungen zufolge ist dieser Harpacticide euryhalin (60\%-20\%); sein Temperaturoptimum liegt bei $22^{\circ}$ bis $25^{\circ} \mathrm{C}$. Ausgehend von einem einzigen befruchteten Weibchen läßt sich in relativ kurzer Zeit in den oben erwähnten Kulturschalen eine einwandfreie Reinkultur heranziehen. Als Futter bewährte sich besonders Trockenhefe (MERck: faex med. sicc. pulv.), die wie folgt präpariert wird: Die Hefe wird mit Aqua destillata zu einem dicken Brei angerührt und anschließend im Trockenschrank bei $80^{\circ} \mathrm{C}$ über Nacht völlig ausgetrocknet. Die harte Masse wird dann im Mörser kleinkörnig zerstampft. Je nach Tierbesatz werden einige Körnchen auf die Oberfläche der Kulturschale gestreut. Die Harpacticiden können offensichtlich nur feste Nahrung aufnehmen, die sie zuvor mit ihren Freßwerkzeugen zerkleinern; pulverisierte Hefe ist demnach nicht geeignet.

\section{Handhabung der Folliculiniden-Kulturen}

Ohne Kenntnis des Lebenszyklus und der of eigentümlichen Verhaltensweisen der freischwimmenden, larvalen Stadien (Schwärmer bzw. Vordertier nach der Teilung) werden sich Mißerfolge bei Kulturversuchen nicht vermeiden lassen. Sämtliche 
Folliculiniden-Schwärmer reagieren mehr oder weniger intensiv thigmotaktisch. Bietet man ihnen eine geeignete, meist leicht aufgerauhte Unterlage an, so führen sie alsbald kriechende Suchbewegungen aus, bis sie sich im günstigen Falle fixieren und den Gehäusebau einleiten. Immer wieder kann man feststellen, daß eine Glasunterlage - sei es die Wandung der Kulturschale oder ein Objektträger - keineswegs eine günstige Ansiedlungsfläche darstellt. Die Schwärmer fixieren sich auf sauberem Glas erst nach einer abnorm verlängerten Schwärmphase, gewissermaßen „unter Zwang“. Nicht selten werden dann auch abnorme Gehäuse gebildet. Es empfiehlt sich daher, die mit Seewasser gefülten Schalen vor der Ubertragung der Schwärmer etwa eine Woche stehen zu lassen. Auf der Schalenwandung entwickelt sich alsbald ein dünner Bakterienfilm. Eine derart präparierte Kulturschale wird von den Schwärmern zweifellos bevorzugt.

Hat man frisches Material gesammelt, so sollten zunächst möglichst viele Tiere aus dem Gehäuse herauspräpariert werden. Man löst das Fußorganell mit einer feinen Stahlnadel vom Gehäuse $a b$ und saugt dann das Tier mit einer dünnen Pipette aus dem Gehäuse. Bei größeren Formen bereitet dies nur wenig Schwierigkeiten. Die präparierten Tiere werden in gesonderten Schalen aufbewahrt. Nach 2 bis 4 Tagen transformieren sie zu Schwärmern (UhLig 1964a) und siedeln sich in der Schale an. Auf diese Weise lassen sich am schnellsten Reinkulturen gewinnen. Kleinere Tiere befreit man weitmöglichst vom Substrat und überträgt sie mit Gehäuse in eine Schale. Viele Tiere transformieren auch dann zu Schwärmern, die abgesammelt und isoliert werden können. Besonders im letzteren Falle (Tiere noch im Gehäuse) empfiehlt sich die Zugabe einiger Harpacticiden.

Bis auf geringe Abweichungen werden die Kulturen der oben genannten vier Arten gleichermaßen behandelt. Zur Züchtung von Klonkulturen wird nach mehrfacher Waschung jeweils ein Schwärmer in eine Boverischale übertragen. Da nur ein gewisser Prozentsatz dieser Einzelkulturen angeht, sollte man gleich mehrere Schalen ansetzen. Nach 6 bis 8 Wochen haben sich die Tiere soweit vermehrt, daß eine Übertragung in eine große Kulturschale möglich ist. Dies geschieht entweder durch Ubersetzen der täglich produzierten Schwärmer in eine neve Schale oder man trennt die ganze Kultur vorsichtig mit einer scharfen Rasierklinge von der Schale ab. Drüdkt man die Klinge fest an die runde Wandung der Schale, und läßt sie, einem Spatel vergleichbar, gleichmäßig von oben nach unten gleiten, so lösen sich die miteinander verklebten Gehäuse vielfach đladenförmig und ohne wesentliche Verletzung der Tiere von der Wandung ab. Bei Uberbesiedlung einer Schale können die Kulturen in der gleichen Weise auf mehrere Schalen verteilt werden. Nach und nach verlassen die Folliculiniden ihre nunmehr schwimmenden Gehäuse und besiedeln wieder die Schalenwandung.

Das Absammeln der Schwärmer wird bei einigen Arten (Metafolliculina andrewsi und der größeren Art von Eufolliculina [Abb. 1a und b]) dank ihres negativ geotaktischen Verhaltens sehr erleichtert. Man schüttet den Inhalt sämtlicher Schalen eines Klons in einen Erlenmeyerkolben und füllt ihn bis zum Rand mit frischem Medium auf. In den entleerten Kulturschalen bleiben die festsitzenden Gehäuse mit den adulten Tieren zurück; man reinigt die Schalen mit der Spritzflasche und füllt frisches Medium auf. Im Erlenmeyerkolben haben sich indessen die Schmutzpartikel abgesetzt, zugleich schwimmen die Schwärmer aktiv nach oben und reichern sich in 


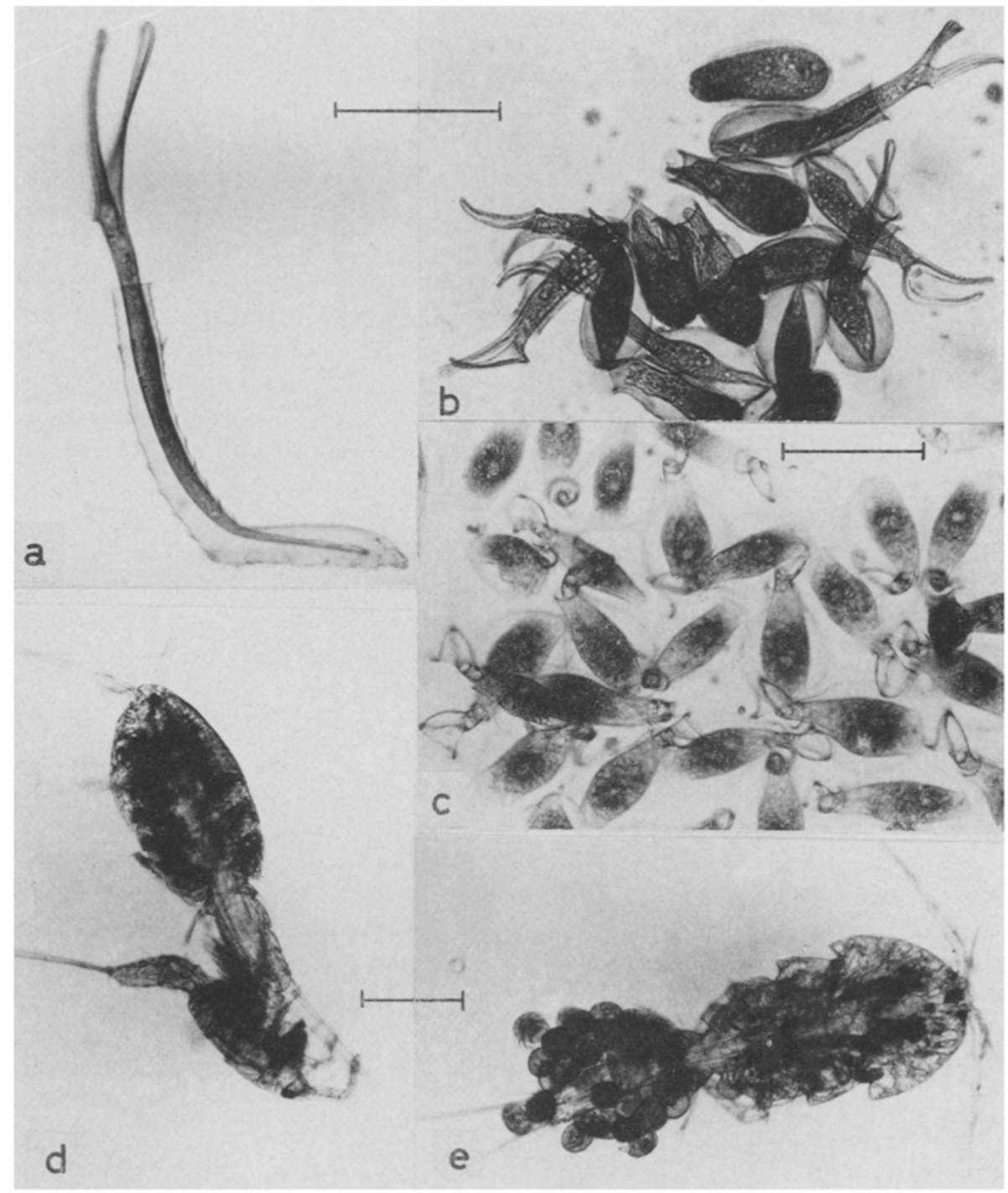

Abb. 1: Lebendaufnahmen der kultivierten Folliculiniden-Spezies sowie des Kulturpartners Tisbe belgolandica (Harpacticidae). (a) Metafolliculina andrewsi. Adultes Tier in gestrecktem Zustand. Gehäusehals mit sekundärer Halsverlängerung; Seitenansicht. (b) Eufolliculina n. sp. Eine kleine Siedlungskolonie der größeren Art (siehe Text). Im Bild oben ein Tier beim Gehäusebau, darunter ein Gehäuse in Seitenansicht. In der Bildmitte einige kontrahierte Tiere. (c) Diafolliculina rotunda. Kolonie-Aussdnitt. Der "Spatelfuß " ist deutlich zu erkennen. Der helle, ein- bis zweigliedrige Makronucleus liegt inmitten einer dunkelgrün etscheinenden Pigmentanhäufung. (d) Tisbe belgolandica n. sp. Männchen und Weibchen bei der Einleitung des Begattungsaktes. Das kleinere Männchen umgreif mit beiden Antennen die Furka des Weibchens. (e) Weibchen mit Eiersack, aus dem gerade die Nauplien ausschlïpfen. Wegen leichter Pressung klaffen die Thoracomeren etwas auseinander. Die gegebenen Maßstäbe entsprechen jeweils 100 u 
der Halsverengung des Kolbens an. Hier können sie leicht mit einer Pipette abgesaugt werden.

Die kleinere Art von Eufolliculina sowie Diafolliculina rotunda (Abb. 1c) zeigen keine negative Geotaxis. Hier müssen die Schwärmer einzeln herauspipettiert oder durch Umschütten in eine neue Schale übertragen werden. Durch stündliches Auszählen der Schwärmer läßt sich die Teilungsrate einer Kultur relativ einfach bestimmen, zugleich erhält man Informationen über eine eventuell gegebene Teilungsperiodizität (UHLIG 1963).

Trotz intensiver, täglicher Reinigungsmanöver" (zum Beispiel kräftiges Ausspritzen) bildet sich in den Schalen nach reichlicher Fütterung und entsprechend zunehmender Besiedlungsdichte eine immer dichter werdende Bodenschicht aus Bakterien, Pilzhyphen und Abfallprodukten der Folliculiniden. Die Tiere werden durch Oberwucherung zunehmend behindert und verharren schließlich in kontrahiertem Zustand im Gehäuse. Die Vermehrungsrate sinkt stark $a b$ und die stagnierenden Kulturen gehen früher oder später ein. Setzt man aber einer stagnierenden. Kultur 20 bis 30 ausgereifte Harpacticiden zu, so beginnen die Folliculiniden innerhalb weniger Stunden wieder "aufzublühen". Die Schmutzschicht wird von den Harpacticiden völlig vertilgt. Erst nach Abtragung dieser Schicht greift Tisbe belgolandica eventuell auch die Gehäuse der Folliculiniden an, nicht aber die 'Tiere selbst. Eine stärkere Beschädigung der Gehäuse wirkt sich allerdings ebenfalls negativ auf die Kultur aus. Es ist darauf zu achten, nur eine, dem jeweiligen Verschmutzungsgrad entsprechende Anzahl Harpacticiden in einer Kultur zu belassen. Sofern eine hohe Teilungsrate der Folliculiniden erzielt werden soll, muß je nach Populationsdichte täglich das Medium gewechselt und gefüttert werden. Anderenfalls genügt es, die Tiere jeden zweiten bis vierten Tag zu versorgen.

Wenn sich in diesen mehrgliedrigen Spezies-Reinkulturen ein ungefähres biologisches Gleichgewicht eingestellt hat, so läßt sich dieses Gleichgewicht durch entsprechende Eingriffe in die resultierende Nahrungskette - Dunaliella und Cryptomonas $\rightarrow$ Follikuliniden $\rightarrow$ Fäkalien $\rightarrow$ Bakterien und Pilze $\rightarrow$ Tisbe b. - wunschgemäß zugunsten der Folliculiniden beeinflussen.

\section{DISKUSSION}

Das beschriebene, mehrgliedrige Kulturverfahren dürtte sich auch auf andere, eulitorale Spezies übertragen lassen. $Z$ weifellos verfügen die litoralen Folliculiniden, in Anpassung an recht extreme Umweltbedingungen, über eine gewisse physiologische Plastizität. Es ist daher verständlich, daß sie sich in gewissen Grenzen auch an die vielfach unnatürlichen Laboratoriumsbedingungen anzupassen vermögen. Andererseits versagt das Verfahren bei Folliculiniden, die relativ konstanten und weniger extremen Umweltfaktoren ausgesetzt sind (Epifauna des Sandgrundes, Formen aus tieferen Meeresbereichen). Zwar können auch diese Formen eine gewisse Zeit in flieBendem Seewasser gehältert werden, eine echte Kultivierung ist jedoch zur Zeit noch nicht möglich. Uberträgt man beispielsweise Muschelschill aus der "Tiefen Rinne" bei Helgoland (Wassertiefe etwa $56 \mathrm{~m}$ ) in ein stehendes Gefäß, so kann man nach ein bis zwei 
Tagen zahlreiche Schwärmer der verschiedensten, auf den Muschelschalen angesiedelten Folliculiniden-Spezies absammeln. Mitunter bauen diese Schwärmer auch auf Glas ein neues Gehäuse, doch ein weiteres Wachstum (Zellteilungen) ist nur selten zu beobachten. Es läßt sich nachweisen, daß diese Schwärmer überwiegend durch Transformation, also Umformung des adulten Tieres zum Schwärmer, gebildet werden. Wie schon ANDrEws (1914, 1923) nachwies, transformieren die Tiere offensichtlich bei Verschlechterung der Umweltbedingungen. Sie verlassen die Gehäuse und siedeln sich in einem günstigeren Milieu wieder an. Die Schwärmphase garantiert damit die Erhaltung und Verbreitung der Art.

An Hand der verfügbaren Folliculiniden-Kulturen gelang es, den Lebenszyklus der bisher am besten untersuchten Art, Metafolliculina andrewsi, weitgehend zu klären (Uhlig 1963, 1964a). Es zeigte sich, daß die adulten, ausdifferenzierten Tiere befähigt sind, den Gehäusehals sekundär zu verlängern (vgl. Abb. 1a). Neben ökologischen Studien werden zur Zeit elektronenmikroskopische Untersuchungen durchgeführt (Uhlig, Komnick \& Wohlfahrt-Bottermann 1965), die unter anderem Aufschluß über die Gehäuse-Sekretion, Kontraktilität, Infraciliatur und cortikale Ultrastruktur geben sollen.

\section{ZUSAMMENFASSUNG}

1. Es wird das Verfahren einer mehrgliedrigen Kultur von vier Arten mariner Flachwasser-Folliculiniden der Gattungen Metafolliculina, Eufolliculina und Diafolliculina beschrieben.

2. Die Folliculiniden werden in Kulturschalen ohne Wasserzirkulation gezüchtet. Saubere Glasflächen sind für die Ansiedlung von Folliculiniden-Schwärmern ungegeeignet. Die Schwärmer bevorzugen Unterlagen, die von einem dünnen Bakterienfilm überzogen sind.

3. Um die zwangsläufig eintretende, allmähliche Verschmutzung der Schalen zu verhindern, wurde ein neu entdeckter Harpacticide - Tisbe belgolandica n. sp. - als Kulturpartner eingeführt.

4. Tisbe helgolandica erwies sich als leicht züchtbares, anspruchloses Laboratoriumstier, das sich dank seiner Ernährungsweise vorzüglich zur Reinhaltung von Kulturen sedentärer, mariner Organismen eignet.

\section{ZITIERTE LITERATUR}

Andrews, E. A., 1914. The bottle-animalcule Folliculina; oecological notes. Biol. Bull. mar. biol. lab., Woods Hole 26, 262-285.

- 1923. Folliculina: Case making, anatomy and transformation. J. Morph. 38, 207-278.

DAs, S. M., 1953. Indian Folliculinidae (Ciliata, Heterotricha). Ann. mag. nat. Hist. (Ser. 12) 6, 235-240.

Dewey, V. C., 1939. Test secretion in two species of Folliculina. Biol. Bull. mar biol. lab., Woods Hole 77, 448-455.

Dons, C., 1924. Metafolliculina nordgardi, n. gen., n. sp. K. norske vidensk. Selsk. Skr. 1, 5-13. 
Fauré-Fremiet, E., 1932. Division et morphogenèse chez Folliculina ampulla O. F. Müller. Bull. biol. Fr. Belg. 66, 78-110.

Hamitton, J. M., 1952. Studies on loricate Ciliophora. 2. Folliculina boltoni Kent. Proc. Iowa Acad. Sci. 58, 469-476.

Hadžr, J., 1938. Beitrag zur Kenntnis der adriatischen Follikuliniden (Inf. Heterotricha). 1. Subfam. Eufolliculininae. Acta adriat. 2, 1-46.

- 1951. Studien über Folliculiniden. Dela slov. Akad. znan. wmet. Hist. nat. Med. 4, 1-390.

Sarhage, H., 1916. Uber die Organisation und den Teilungsvorgang des Flaschentierchens (Folliculina ampulla). Arch. Protistenk. 37, 139-174.

Utt.IG, G., 1963. Untersuchungen über die Folliculiniden (Ciliata; Heterotricha) der Deutschen Bucht. Veröff. Inst. Meeresforsch. Bremerhaven, Sonderbd., 115-121.

- 1964a. Der Gehäusebau bei Metafolliculina andrezesi (Ciliata; Heterotricha). Zool. Anz. (Suppl. Bd.) 27, 498-507.

- 1964b. Die Folliculiniden der Deutschen Bucht. 1. Magnifolliculina n. g., eine interessante Formengruppe der Epifauna des Sandgrundes. Helgoländer wiss. Meeresunters. 11, 92-109.

-, Komnick, H. \& Wohlfarth-Bottermann, K. E., 1965. Intrazelluläre Zellzotten in Nahrungsvaknolen von Ciliaten. Helgoländer wiss. Meeresunters. 12, 61-77.

- \& Noodr, W., 1965. Tisbe helgolandica n. sp. aus dem Seewasser-Freibad Helgoland. Kieler Meeresforsch. (im Druck).

WrIGHT, T. S., 1859. Description of new Protozoa. Edinb. new. phil. J. 10, 97-101. 